\title{
Efficacy and safety of HX 110-A and HX 110-B in promoting respiratory health: protocol for an 8-week, randomized, double-blind, parallel group, placebo-controlled trial
}

\author{
Jung-Kyu Lee ${ }^{1 \wedge}$, Bumjo $\mathrm{Oh}^{2}$, Hyun Woo Lee ${ }^{1}$, Seo-Young Yoon ${ }^{1}$, Tae Yun Park ${ }^{1}$, Eun Young Heo ${ }^{1}$, \\ Deog Kyeom Kim ${ }^{1}$
}

${ }^{1}$ Division of Pulmonary and Critical Care Medicine, Department of Internal Medicine, ${ }^{2}$ Department of Family Medicine, Seoul Metropolitan Government-Seoul National University Boramae Medical Center, Republic of Korea

Contributions: (I) Conception and design: All authors; (II) Administrative support: JK Lee, DK Kim; (III) Provision of study materials or patients: All authors; (IV) Collection and assembly of data: All authors; (V) Data analysis and interpretation: All authors; (VI) Manuscript writing: JK Lee, DK Kim; (VII) Final approval of manuscript: All authors.

Correspondence to: Deog Kyeom Kim, MD, PhD. Division of Pulmonary and Critical Care Medicine, Department of Internal Medicine, Seoul Metropolitan Government-Seoul National University Boramae Medical Center, \#395 Shindaebang-2-Dong, Dongjak-Gu, Seoul, 07061, Republic of Korea. Email: kimdkmd@gmail.com.

Background: HX110-A and HX110-B are compound extracts based on radix adenophorae and rhizoma dioscoreae, respectively, which have anti-inflammatory activity. There are limited data on whether they may help improve respiratory conditions including lung function. Therefore, in this trial, we will evaluate the effectiveness and safety of the use of HX110-A and HX110-B for the treatment of respiratory health in adults with mild respiratory symptoms.

Methods: This will be an 8-week, randomized, double-blind, parallel group, placebo-controlled trial with three arms. Adults more than 40 years old with persistent respiratory symptoms will be enrolled. Patients with definite respiratory disease or with a history of recent intake of antioxidants or anti-inflammatory agents will be excluded. Study subjects will be assigned at a 1:1:1 ratio into the following three arms: controls, experimental group 1 (HX110-A), and experimental group 2 (HX110-B). Control or experimental foods will be administered for 8 weeks, and follow-up will be up to 12 weeks. The primary outcome will be total antioxidant capacity. Secondary outcomes will be inflammatory indexes, respiratory symptoms, lung function, quality of life, and fatigue level. Safety outcomes will be assessed by monitoring adverse events and vital signs, and through clinical pathology tests.

Discussion: This trial will reveal the effectiveness and safety of HX110-A and/or HX110-B for medical purposes in adults with respiratory symptoms. The results should clarify if active intake of specific foods with these functional compounds may promote respiratory health in adults without definite respiratory disease.

Trial registration: Clinical Research Information Service, KCT0003614. Registered 12 May 2019 (Respectively registered, https://cris.nih.go.kr/cris/en/search/search_result_st01.jsp?seq=13364).

Keywords: Dietary supplements; antioxidants; health promotion; randomised controlled trial

Submitted Jun 02, 2020. Accepted for publication Aug 12, 2020.

doi: 10.21037/apm-20-1214

View this article at: http://dx.doi.org/10.21037/apm-20-1214

\footnotetext{
$\wedge$ ORCID: 0000-0001-5060-7255.
} 


\section{Introduction}

Although respiratory disease is prevalent and may lead to high mortality, it tends to be detected in advanced stages of disease due to low awareness $(1,2)$. As a result, current therapeutic trials on respiratory diseases often show limited effect improving respiratory health (3). This is why a strategy to promote respiratory health status and prevent the progression of disease is important $(4,5)$. To this end, it would be useful to develop effective and safe functional raw materials that may help prevent aging of respiratory organs and improve damage to organs so that they do not fall into the irreversible stages of disease.

HX110-A and HX110-B are compound extracts of radix adenophorae and rhizoma dioscoreae, respectively, traditionally used for respiratory diseases due to their mucolytic, expectorant, and anti-inflammatory activities. Radix adenophorae extract (RAE) enhances immune function and protects against exogenous pathogens by activating macrophages (6). It also reduces tissue eosinophilia and bronchial hyperresponsiveness, which suggests that it may play a positive role in patients with respiratory symptoms of airway disease (7). In a study that used an animal model of chronic obstructive pulmonary disease (COPD), RAE inhibited cigarette smoke-induced pulmonary inflammation and mucin hypersecretion (8). Rhizoma dioscoreae extract (RDE) produces immunomodulatory effects by inhibiting pro-inflammatory cytokines $(9,10)$.

Despite these positive findings, there are limited data on whether HX110-A and HX110-B may improve symptoms and prevent further progression of respiratory disease in adults. Therefore, we plan to evaluate the effectiveness and safety of these compounds on respiratory health in adults with mild respiratory symptoms.

\section{Methods}

\section{Study setting and design}

This randomized controlled study will be conducted at the Seoul Metropolitan Government-Seoul National University Boramae Medical Center, a tertiary referral hospital in South Korea. The flow diagram of the trial is presented in Figure 1.

This will be an 8 -week, randomized, double-blind, parallel group, placebo-controlled trial with three arms, as summarized below:

(I) Arm 1 (control arm): placebo; lactose mixed powder; $500 \mathrm{mg}$ tablet twice a day

(II) Arm 2 (experimental group 1): HX110-A; RAE (root of Adenophora stricta Miq), seed of Brassica juncea Czern. et Cosson, leaf and stem of Lonicera japonica Thunberg; $500 \mathrm{mg}$ tablet (containing HX $110-\mathrm{A} 150 \mathrm{mg} /$ tablet) twice a day

(III) Arm 3 (experimental group 2): HX110-B; RDE (rhizome of Dioscorea batatas Decaisne), grass of Taraxacum officinale Weber, and flower stalk of Schizonepeta tenuifolia Briq.; $500 \mathrm{mg}$ tablet (containing HX 110-B $150 \mathrm{mg} /$ tablet) twice a day

Element composition of these study medications enlists in Table 1. The study timeline is presented in Table 2. Study subjects who voluntarily consent to the study at visit 1 (week 0 ) will be judged for eligibility according to inclusion and exclusion criteria. Selected subjects will be randomly assigned to study groups, and tested for functional biomarkers after ingesting the experimental or control foods for 8 weeks. Then they will stop taking the foods for the next 4 weeks and receive a functional evaluation at visit 5 (week 12). First enrollment of study participant was 7 February 2019. This study was retrospectively registered in Clinical Research Information Service on 12 March 2019 (CRIS registration number: KCT0003614; URL: https://cris.nih.go.kr/cris/en/search/search_result_st01. jsp? seq=13364). The study was conducted in accordance with the Declaration of Helsinki (as revised in 2013). This trial received ethical approval from the Institutional Review Board of the Seoul Metropolitan Government-Seoul National University Boramae Medical Center (IRB no. 302018-90). Written informed consent will be obtained from all study participants.

\section{Set-up of dosage}

In a previous study that investigated the effects of HX110-A and HX110-B on respiratory health in animal models of pancreatic porcine elastase-induced emphysema, mice were orally administered $100 \mathrm{mg} / \mathrm{kg}$ per day for 3 weeks. Therefore, in this study, the effective intake of HX110-A and HX110-B will be set to $100 \mathrm{mg} / \mathrm{kg}$ per day, and the average daily intake will be $300 \mathrm{mg}$ assuming that the average body weight of an adult is $60 \mathrm{~kg}$ (11).

\section{Eligibility criteria}

The inclusion criteria will be as follows: men and women more than 40 years old, with persistent respiratory 

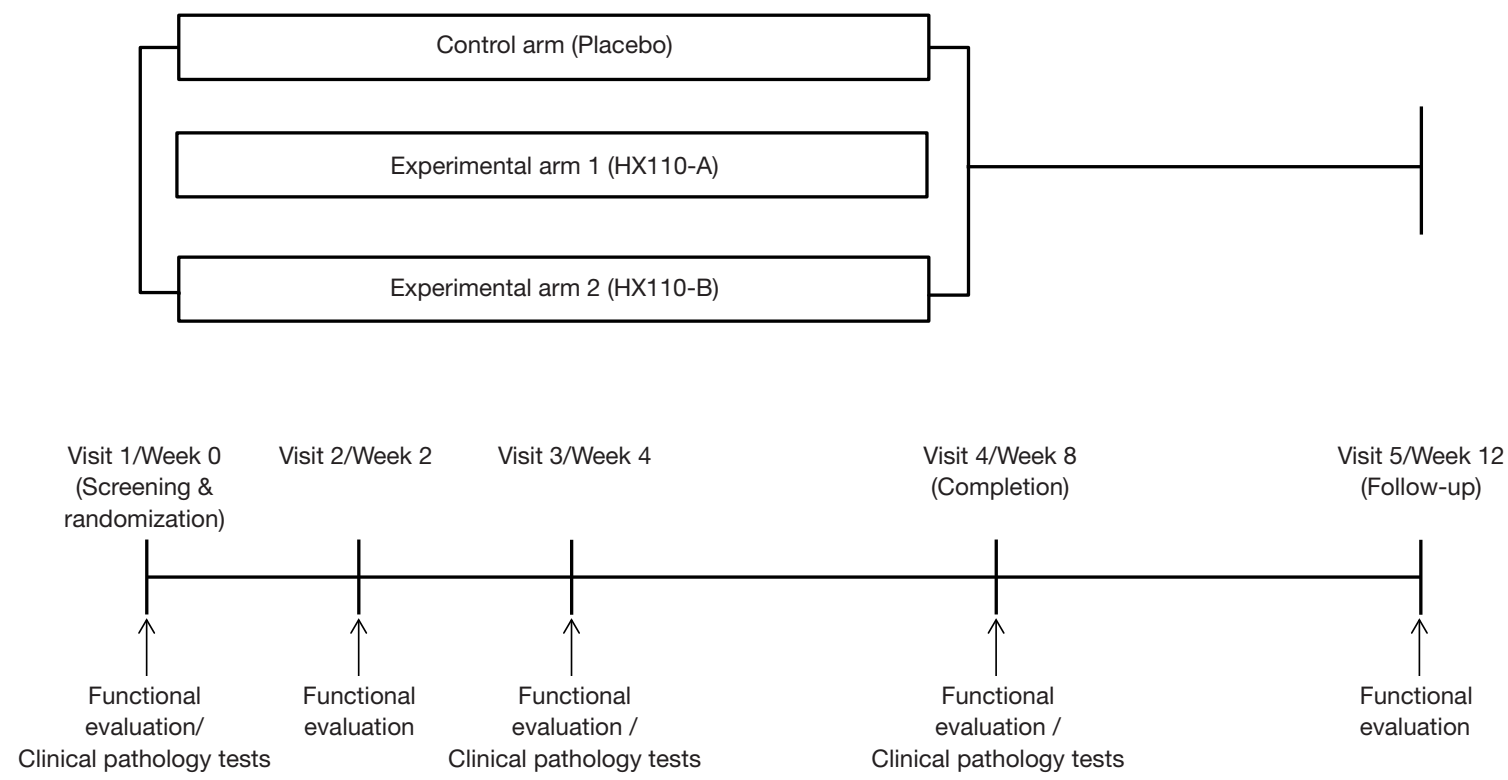

Figure 1 Flow diagram of the trial.

Table 1 Element composition of the study medication

\begin{tabular}{lccc}
\hline Element & Placebo (\%) & Experimental food 1 (\%) & Experimental food 2 (\%) \\
\hline HX110-A powder (extract : maltodextrine = 3:1) & 0.0 & 40.0 & 0.0 \\
HX110-B powder (extract : maltodextrine = 3:1) & 0.0 & 0.0 & 40.0 \\
Microcrystalline cellulose & 29.12 & 13.73 & 40.0 \\
Lactose mixed powder & 43.0 & 0.90 & 13.73 \\
Hydroxypropyl cellulose & 22.51 & 1.97 & 0.90 \\
Hydroxypropylmethyl cellulose & 1.97 & 1.0 & 1.97 \\
Silicon dioxide & 1.0 & 1.0 & 1.0 \\
Magnesium stearate & 1.0 & 0.65 & 1.0 \\
Titanium dioxide & 0.65 & 0.70 & 0.65 \\
Caramel pigment & 0.70 & 0.05 & 0.05 \\
Glycerin fatty acid ester & 0.05 & 100.0 & 100.0 \\
Total & 100.0 & 0 & \\
\hline
\end{tabular}

symptoms including cough and sputum, and who can follow the dietary and lifestyle guidelines during the study period.

Patients will be excluded if they meet any of the following: definite respiratory disease, including acute bronchitis, chronic bronchitis, pneumonia, tuberculosis, asthma, COPD, and lung cancer; diabetes or cardiovascular diseases except hypertension; taking or having taken antioxidants or anti-inflammatory drugs within the past 6 weeks; serum levels of creatinine $\geq 2 \mathrm{mg} / \mathrm{dL}$ or end-stage renal disease; serum levels of total bilirubin $\geq 2 \mathrm{mg} / \mathrm{dL}$, or aspartate aminotransferase or alanine aminotransferase levels of $\geq 60$ IU/L; uncontrolled psychological disease or alcoholic; taking health-promoting foods with antioxidant activity (Recommended Food Score $>36$ ); body mass index 
Table 2 Study timeline

\begin{tabular}{|c|c|c|c|c|c|}
\hline \multirow{2}{*}{ Variables/assessed items } & \multicolumn{4}{|c|}{ Intervention period } & \multirow{2}{*}{$\begin{array}{l}\text { Follow-up } \\
\text { (week 12) }\end{array}$} \\
\hline & Week 0 & Week 2 & Week 4 & Week 8 & \\
\hline Visit number & 1 & 2 & 3 & 4 & 5 \\
\hline Consent & $\sqrt{ }$ & & & & \\
\hline Baseline characteristics $^{1}$ & $\sqrt{ }$ & & & & \\
\hline Medical history & $\sqrt{ }$ & & & & \\
\hline Physical examination & $\sqrt{ }$ & $\sqrt{ }$ & $\sqrt{ }$ & $\sqrt{ }$ & $\sqrt{ }$ \\
\hline Chest X-ray & $\sqrt{ }$ & & & $\sqrt{ }$ & $\sqrt{ }$ \\
\hline Drug and functional health food intake & $\sqrt{ }$ & $\sqrt{ }$ & $\sqrt{ }$ & $\sqrt{ }$ & $\sqrt{ }$ \\
\hline Screening tests ${ }^{2}$ & $\sqrt{ }$ & & & & \\
\hline Randomization & $\sqrt{ }$ & & & & \\
\hline Smoking history & $\sqrt{ }$ & $\sqrt{ }$ & $\sqrt{ }$ & $\sqrt{ }$ & $\sqrt{ }$ \\
\hline Drinking history & $\sqrt{ }$ & & & & \\
\hline Physical activity history (international physical activity questionnaire) & $\sqrt{ }$ & & & & \\
\hline Functional evaluation $^{4}$ & $\sqrt{ }$ & $\sqrt{ }$ & $\sqrt{ }$ & $\sqrt{ }$ & $\sqrt{ }$ \\
\hline Education about dietary and life habits & $\sqrt{ }$ & $\sqrt{ }$ & $\sqrt{ }$ & $\sqrt{ }$ & \\
\hline Dietary intake ${ }^{5}$ & $\sqrt{ }$ & & & $\sqrt{ }$ & $\sqrt{ }$ \\
\hline Monitoring of dietary and life habits ${ }^{6}$ & & $\sqrt{ }$ & $\sqrt{ }$ & $\sqrt{ }$ & $\sqrt{ }$ \\
\hline
\end{tabular}

${ }^{1}$, demographic surveys (gender, date of birth, age, menstruation, and amenorrhea) and history of colds (number of colds in the past year and average duration of a cold). ${ }^{2}$, measure the following after $12 \mathrm{~h}$ fasting. Blood test: creatinine, total bilirubin, aspartate aminotransferase, alanine aminotransferase (if there is a test result within 4 weeks, it can be omitted); Pregnancy test, urinary test should be performed only for women of childbearing age except for those subjects in menopause (amenorrhea more than 24 months); Respiratory symptoms, BCSS. ${ }^{3}$, height is measured only at visit 1 (week 0 ). ${ }^{4}$, measure the following after a $12 \mathrm{~h}$ fast. Antioxidant activity: TAC (weeks $0,2,4$, 8, and 12); Inflammatory index: SRAGE, SP-D, CC-16, complete blood count/differential count, hsCRP (weeks 0, 2, 4, 8, and 12), FeNO (weeks 0, 8, and 12); Respiratory symptoms: BCSS, CASA-Q, COAT, VAS (weeks 0, 2, 4, 8, and 12); Lung function: FEV1, FVC, DLCO (weeks 0, 8, and 12); Quality of life: EQ-5D (weeks 0, 4, 8, and 12); Fatigue: FSS (weeks 0, 2, 4, 8, and 12). *BCSS and VAS are written daily from visit 1 (week 0 ) and verified at visit 2 (week 2). ${ }^{5}$, the E-diary (a dietary and lifestyle research program available on a smartphone or computer) will be used in visits 1 (week 0), 4 (week 8), and 5 (week 12) to reflect the usual dietary intake. If a study subject has difficulty using a smartphone or computer, the investigator will ask the subject to write daily records of dietary and life habits. ${ }^{6}$, use E-diary to monitor dietary and lifestyle guidelines and recheck for each visit. If study subject has difficulty using a smartphone or computer, the investigator asks the subject to write daily records of dietary and life habits, and checks them at each visit. ${ }^{7}$, measure the following after a $12 \mathrm{~h}$ fast. However, the items included in the functional evaluation among the hematological tests will not be measured separately and can be omitted if there are test results within 4 weeks. Hematological tests: complete blood count, differential count (neutrophils, eosinophils, basophils, lymphocytes, monocytes), erythrocyte sedimentation rate; Blood chemistry tests: aspartate aminotransferase, alanine aminotransferase, $\gamma$-glutamyl transferase, alkaline phosphatase, blood urea nitrogen, creatinine, total bilirubin, uric acid, total protein, albumin, glucose, and estimated glomerular filtration rate; Urine tests: $\mathrm{pH}$, albumin, glucose, ketone, blood, urobilinogen, bilirubin, nitrite, leukocytes, and specific gravity. ${ }^{8}$, monitor compliance using the E-diary and reconfirm at each visit. If study subject has difficulty using a smartphone or computer, the investigator asks the subject to write daily records of dietary and life habits, and checks them at each visit. 
$\geq 35 \mathrm{~kg} / \mathrm{m}^{2}$; history of hypersensitivity for testing substance; involved in other clinical trials within 1 month; and pregnancy or lactating status.

\section{Randomization}

A random number will be assigned during visit 1 (week 0 ) in the order in which the subject is enrolled according to the inclusion and exclusion criteria. Subjects will be assigned to the study group, based on a computer-generated random list prepared by a third party independent of the study before the study begins. Study participants will be allocated to the control group, the experimental group 1 (HX110-A), and the experimental group 2 (HX110-B) at a 1:1:1 ratio using a block randomization method. The study will be double-blind and group assignments will not be disclosed to investigators or subjects until the end of the study.

\section{Food distribution and retrieval}

Experimental or control foods will be distributed to randomized subjects during visit 1 (week 0), 2 (week 2), and 3 (week 4). Subjects will be instructed to bring the remaining experimental or control food after visit 2 (week 2), 3 (week 4) and 4 (week 8), and the researchers will reconfirm the remaining amount of experimental or control food. A compliance rate of $80 \%$ or more will be considered good compliance.

\section{Fustification of sample size}

The hypothesis of this study is that the use of HX110-A or HX110-B will improve antioxidant and inflammatory indices and respiratory symptoms. Therefore, a primary comparison will be conducted between the control arm and experimental arm 1 (HX100-A).

\section{Hypotheses}

Ho (null hypothesis): $\mu \mathrm{t}=\mu \mathrm{c}$ (the respiratory health index after 12 weeks of treatment will not be different between the control arm and experimental arm).

H1 (alternative hypothesis): $\mu \mathrm{t} \neq \mu \mathrm{c}$ (the respiratory health index after 12 weeks of treatment will be different between the control arm and experimental arm).

\section{Assumptions}

Previous studies have reported differences in total antioxidant capacity changes of $0.09 \pm 0.10 \mathrm{mmol} / \mathrm{L}(12)$, and forced expiratory volume in $1 \mathrm{~s}$ (FEV1), FEV25-75\%, peak expiratory flow variability, and fractional expired nitric oxide $(\mathrm{FeNO})$ values of $7.70 \% \pm 8.50 \%, 0.40 \pm 0.45 \mathrm{~L} / \mathrm{s}$, $5.80 \pm 6.30$, and $4.60 \pm 5.00 \mathrm{ppb}$, respectively, after control or test food intake (13). Hence, the sample size per arm for the proposed study was calculated as 20 (60 in total), based on a power of $80 \%$ and an $\alpha=0.05$ level of significance. Considering a dropout rate of $20 \%$, the final number of subjects per arm would be 25 (75 in total).

\section{Statistical analyses}

Statistical analyses will be performed using intention-totreat or per-protocol analyses. Tests of normality will be performed to evaluate the data distribution. Data that are not normally distributed will be converted to achieve a normal distribution and then analyzed using a parametric method, or will be analyzed with a non-parametric method. Each test group of continuous variables will be compared to the control group using Student's t-test, analysis of covariance, and the Wilcoxon rank-sum test. Comparison between groups according to changes in time will be analyzed using a mixed model, and analysis of variance and the Kruskal-Wallis test may also be performed among the three groups. Intra-group comparisons between before and after the intervention will be analyzed using the paired t-test and Wilcoxon signed rank test. Correlations among variables will be analyzed using Pearson's correlation and regression analyses. Categorical data will be analyzed using the chi-square test or Fisher's exact test. A two-sided test will be considered significant when the $\mathrm{P}$ value $<0.05$.

\section{Efficacy outcomes}

The assessment of efficacy will be based on a biomarker and functional evaluation. The primary outcome will be the total antioxidant capacity. Secondary outcomes will be as follows: (I) inflammatory indices: soluble forms of receptor of advanced glycation end products (sRAGE), surfactant protein D (SP-D), club cell secretory protein 16 (CC-16), high sensitivity C-reactive protein (hsCRP), and FeNO; (II) respiratory symptoms: Breathlessness, Cough, and Sputum Scale (BCSS), Cough And Sputum Assessment Questionnaire (CASA-Q), Cough Assessment Test (COAT), and visual analogue scale (VAS) score; (III) lung function: FEV1, forced vital capacity, diffusion capacity; (IV) quality of life: EuroQol 5 dimension scale (EQ-5D); and (V) fatigue: Fatigue Severity Score (FSS). Antioxidant capacity 
and inflammatory markers will be estimated after a 12-hour fast.

\section{Safety assessment}

The safety of the study will be assessed by monitoring adverse events and vital signs, and using the clinical pathology tests to test blood and urine samples. Subjects will be asked to voluntarily report any adverse events. During the study period, the investigators will check for adverse reactions through interviews and questionnaires. The investigation of adverse events will include the date of development and disappearance, the extent and consequences of the reaction, the actions taken in relation to the test food, the causal relationship with the test food, and treatment.

Clinical pathology tests will be performed at visits 1 (week 0), 3 (week 4), and 4 (8 week), after a 12-hour fast. However, the items included in the functional evaluation among the hematological tests will not be measured separately and may be omitted if there are test results within 4 weeks. Specific clinical pathology items are described in the footnotes of Table 2 .

\section{Education on and investigation of diet and lifestyle}

During the study period, study subjects will be educated to follow the guidelines below, but otherwise to maintain their usual diet and lifestyle: (I) no ingestion of test ingredients or foods containing these ingredients; (II) limited fruit and vegetable intake (fruit and fruit juice $\leq 2$ servings/day, vegetables $\leq 6$ servings/day); (III) no ingestion of seaweed and vegetable pellets or extracts of onion juice, pumpkin juice, pear juice, and grape juice; (IV) no intake of dietary supplements or herbal medication, or drugs specified in the exclusion criteria; (V) no smoking; (VI) no hard exercise up to the day before the visit.

From visit 1 (week 0 ) to visit 4 (week 8), the investigator will educate subjects on how to use an e-diary during the study. At visits 1 (week 0), 4 (8 week), and 5 (week 12), subjects will complete the diary for daily dietary intake and compliance with daily dietary and lifestyle guidelines. If it is difficult for the subject to use a smartphone or computer, the investigator will ask the subject to write daily records of dietary and life habits, and check them at each visit. The investigator will monitor subjects' adherence to dietary and lifestyle guidelines from visit 1 (week 0 ) until the end of the study.

\section{Study discontinuation, withdrawal, and protocol violation}

The following cases may be used to suspend or drop a study subject: (I) if a severe adverse event occurs, (II) a subject or legal representative wants to stop participating, (III) if a researcher or subject violates the study plan, (IV) if a subject withdraws consent to participate, (V) if a subject cannot be followed up, and (VI) when a researcher judges that the progress of the study is inappropriate.

Study subjects will consume the distributed experimental or control foods in accordance with the guidelines. The investigator will take appropriate measures to ensure compliance with the food intake and visit plan, such as a written notice of the next visit or telephone monitoring. When a violation of the study plan occurs, the subject will be dropped from the study if the violation has a significant impact on the subject's safety and study results. For other minor violations, the extent and reason for the violation should be accurately documented and the impact of the violation on the study should be assessed in the analyses of the results.

\section{Data collection and monitoring}

This study will be designed so that the study subjects can input data through an e-diary, and the researcher can check the contents and progress. The e-diary is a program designed to allow study subjects to directly record their diet and lifestyle during the study using a smartphone or computer and has a function for the researcher to monitor the subject's compliance with the study guidelines.

\section{Supervision of the trial}

Staff will monitor the progress of the research to protect the rights and welfare of the subjects, ensure that the reported research data are accurate and verifiable against the supporting documentation, and ensure that the research is carried out in accordance with the approved plan. Monitoring will be by regular visit to the research institute and by telephone. The monitoring staff will check the original subject records, the management records of the experimental and control foods, the archived data files, and the progress of the study, and will consult with the researcher if there is a problem. The staff will monitor 18 times (immediately after registration starts, every $10 \%$ enrollment from $10 \%$ to $100 \%$, every $10 \%$ completion from $50 \%$ to $100 \%$, and within 2 weeks after completion) 
and report these results to the monitoring officer.

\section{Confidentiality}

Records confirming the subject's identity will be kept confidential. Monitoring staff and officers involved in the study may access subject records for the purpose of monitoring, reviewing, or managing the progress of the study, but such information will also be kept confidential. All documents related to the study, such as case records, will be recorded and distinguished using a subject identification code, not subject name. The collected information will be kept in the document storage room of the research institute for 3 years after the end of the study, and will be discarded after 3 years according to the standards and procedures prescribed by the Personal Information Protection Act. During or after the study, research data, including medical records, may be reviewed to verify the conduct of the study and the reliability of the data, but this access is limited to the extent to which confidentiality of each subject's identity is protected.

\section{Discussion}

Patients with chronic respiratory disease, such as COPD, experience pathological changes in the airway and lung function at an early stage (14). Recent studies have shown that patients with early-stage COPD may have a faster decline in lung function than those with more advanced COPD, and may also experience chronic respiratory symptoms and acute exacerbations $(15,16)$. Even symptomatic current or former smokers with preserved lung function, who do not meet the COPD criteria, have exacerbations and limitations in activities (17). This is why early detection and active treatment before respiratory disease advances is important. It has recently been reported that inhaled long-acting muscarinic antagonists reduce the annual FEV1 decline even in patients with early COPD (5), and large-scale studies have indicated that active treatment with inhalers reduces the risk for mortality and acute exacerbation in patients with mild- to moderate-stage COPD (18-20).

Our proposed study will determine whether active intervention can have beneficial effects on the respiratory health of adults who have respiratory symptoms but do not have a definite respiratory disease. Few studies have investigated treatment effectiveness and safety among such subjects. In particular, the key point is whether respiratory health will benefit from foods that are expected to have less adverse reactions than drugs. Medicine diets have traditionally existed in various forms, and there have been attempts to apply them to patients with chronic respiratory disease $(21,22)$.

We note the anti-inflammatory and immune-modulating effects of the raw materials contained in HX100-A or HX110-B. Data on the anti-inflammatory effects of RAE and RDE are mentioned above. In addition, the compounds in Taraxacum officinale and Lonicera japonica have antiinflammatory effects by inhibiting expression of proinflammatory cytokines and chemokines at the mRNA and protein levels (23). Inhalable microparticles of Lonicera japonica decrease the levels of tumor necrosis factor- $\alpha$ and interleukin-6 in bronchoalveolar fluid and inflammatory cells in peripheral blood, induce recovery of elastin and collagen distribution, and reduce caspase- 3 expression in lung tissue (24). Schizonepeta tenuifolia suppresses immunoglobulin E-induced allergic reactions at the cellular level, and ameliorates inflammatory cytokines and eosinophilic inflammation $(25,26)$. These results suggest that HX110 can prevent the development of chronic airway disease that occurs as chronic inflammation persists.

This study is designed to evaluate whether HX110, a compound in food for medical purposes, has an antiinflammatory function and improves respiratory conditions in adults with respiratory symptoms. The results will clarify if active intake of specific foods with functional materials may promote respiratory health in adults without definite respiratory disease.

\section{Acknowledgments}

We would like to thank Helixmith Co. Ltd., Seoul, Republic of Korea, for their financial support for clinical research expenses and supply of investigational products and laboratory tests. We thank Young Ran Hwang, R.N., and Myung Ja Lee, R.N. for their support.

Funding: This study was supported by a grant from the Helixmith Co., Ltd. The funding bodies have no role in study design, collection, management, analysis, interpretation of data, writing the manuscript or decision to submit the report for publication.

\section{Footnote}

Conflicts of Interest: All authors have completed the ICMJE uniform disclosure form (available at http://dx.doi. 
org/10.21037/apm-20-1214). The authors have no conflicts of interest to declare.

Ethical Statement: The authors are accountable for all aspects of the work in ensuring that questions related to the accuracy or integrity of any part of the work are appropriately investigated and resolved. The study was conducted in accordance with the Declaration of Helsinki (as revised in 2013). This trial received ethical approval from the Institutional Review Board of the Seoul Metropolitan Government-Seoul National University Boramae Medical Center (IRB no. 30-2018-90), and was registered with Clinical Research Information Service (KCT0003614, https://cris.nih.go.kr/cris/en/search/search_result_st01. jsp? seq=13364) on 12 March 2019. Written informed consent will be obtained from all study participants.

Open Access Statement: This is an Open Access article distributed in accordance with the Creative Commons Attribution-NonCommercial-NoDerivs 4.0 International License (CC BY-NC-ND 4.0), which permits the noncommercial replication and distribution of the article with the strict proviso that no changes or edits are made and the original work is properly cited (including links to both the formal publication through the relevant DOI and the license). See: https://creativecommons.org/licenses/by-nc-nd/4.0/.

\section{References}

1. Seo JY, Hwang YI, Mun SY, et al. Awareness of COPD in a high risk Korean population. Yonsei Med J 2015;56:362-7.

2. Hogg JC, Chu F, Utokaparch S, et al. The nature of smallairway obstruction in chronic obstructive pulmonary disease. N Engl J Med 2004;350:2645-53.

3. Tantucci C, Modina D. Lung function decline in COPD. Int J Chron Obstruct Pulmon Dis 2012;7:95-9.

4. van den Boom G, Rutten-van Molken MP, Molema $\mathrm{J}$, et al. The cost effectiveness of early treatment with fluticasone propionate 250 microg twice a day in subjects with obstructive airway disease. Results of the DIMCA program. Am J Respir Crit Care Med 2001;164:2057-66.

5. Zhou Y, Zhong NS, Li X, et al. Tiotropium in Early-Stage Chronic Obstructive Pulmonary Disease. N Engl J Med 2017;377:923-35.

6. Li JW, Liu Y, Li BH, et al. A polysaccharide purified from Radix Adenophorae promotes cell activation and proinflammatory cytokine production in murine RAW264.7 macrophages. Chin J Nat Med 2016;14:370-6.

7. Roh SS, Kim SH, Lee YC, et al. Effects of radix adenophorae and cyclosporine A on an OVA-induced murine model of asthma by suppressing to T cells activity, eosinophilia, and bronchial hyperresponsiveness. Mediators Inflamm 2008;2008:781425.

8. Seo HS, Lee HJ, Lee CJ. Effect of Pyunkang-tang on Inflammatory Aspects of Chronic Obstructive Pulmonary Disease in a Rat Model. Nat Prod Sci 2019;25:103-10.

9. Kim S, Shin S, Hyun B, et al. Immunomodulatory Effects of Dioscoreae Rhizome Against Inflammation through Suppressed Production of Cytokines Via Inhibition of the NF-kappaB Pathway. Immune Netw 2012;12:181-8.

10. Kim MJ, Kim HN, Kang KS, et al. Methanol extract of Dioscoreae Rhizoma inhibits pro-inflammatory cytokines and mediators in the synoviocytes of rheumatoid arthritis. Int Immunopharmacol 2004;4:1489-97.

11. FDA. FDA Guidance for Industry - Estimating the maximum safe starting dose in initial clinical trials for therapeutics in adult healthy volunteers. 2005.

12. Ghorbanihaghjo A, Safa J, Alizadeh S, et al. Protective effect of fish oil supplementation on DNA damage induced by cigarette smoking. J Health Popul Nutr 2013;31:343-9.

13. Salem AM, Bamosa AO, Qutub HO, et al. Effect of Nigella sativa supplementation on lung function and inflammatory mediatorsin partly controlled asthma: a randomized controlled trial. Ann Saudi Med 2017;37:64-71.

14. Rodríguez-Roisin R, Drakulovic M, Rodríguez DA, et al. Ventilation-perfusion imbalance and chronic obstructive pulmonary disease staging severity. J Appl Physiol (1985) 2009;106:1902-8.

15. Dransfield MT, Kunisaki KM, Strand MJ, et al. Acute Exacerbations and Lung Function Loss in Smokers with and without Chronic Obstructive Pulmonary Disease. Am J Respir Crit Care Med 2017;195:324-30.

16. Çolak Y, Afzal S, Nordestgaard BG, et al. Prevalence, Characteristics, and Prognosis of Early Chronic Obstructive Pulmonary Disease. The Copenhagen General Population Study. Am J Respir Crit Care Med 2020;201:671-80.

17. Woodruff PG, Barr RG, Bleecker E, et al. Clinical Significance of Symptoms in Smokers with Preserved Pulmonary Function. N Engl J Med 2016;374:1811-21.

18. Decramer M, Celli B, Kesten S, et al. Effect of tiotropium on outcomes in patients with moderate chronic obstructive pulmonary disease (UPLIFT): a prespecified subgroup analysis of a randomised controlled trial. Lancet 2009;374:1171-8. 
19. Jenkins CR, Jones PW, Calverley PM, et al. Efficacy of salmeterol/fluticasone propionate by GOLD stage of chronic obstructive pulmonary disease: analysis from the randomised, placebo-controlled TORCH study. Respir Res 2009;10:59.

20. Tashkin DP, Celli BR, Decramer M, et al. Efficacy of tiotropium in COPD patients with FEV1 $>/=$ $60 \%$ participating in the UPLIFT(R) trial. COPD 2012;9:289-96.

21. Zhao YL, Song HR, Fei JX, et al. The effects of Chinese yam-epimedium mixture on respiratory function and quality of life in patients with chronic obstructive pulmonary disease. J Tradit Chin Med 2012;32:203-7.

22. Alamgeer, Younis $\mathrm{W}$, Asif $\mathrm{H}$, et al. Traditional medicinal plants used for respiratory disorders in Pakistan: a review of the ethno-medicinal and pharmacological evidence.

Cite this article as: Lee JK, Oh B, Lee HW, Yoon SY, Park TY, Heo EY, Kim DK. Efficacy and safety of HX 110-A and HX 110-B in promoting respiratory health: protocol for an 8-week, randomized, double-blind, parallel group, placebocontrolled trial. Ann Palliat Med 2020;9(5):3584-3592. doi: 10.21037/ apm-20-1214
Chin Med 2018;13:48.

23. Lee BS, Kim HY, Kim JJ, et al. The Inhibitory Effects of Haeyeol-tang in Expression of Pro-inflammatory Cytokine on LPS-stimulated THP-1 Cells. Korean J Orient Int Med 2008;29:334-47.

24. Park YC, Jin M, Kim SH, et al. Effects of inhalable microparticle of flower of Lonicera japonica in a mouse model of COPD. J Ethnopharmacol 2014;151:123-30.

25. Lin YH, Chen HY, Chiu JC, et al. Immunomodulation Effects of Schizonepeta tenuifolia Briq. on the IgEInduced Allergic Model of RBL-2H3 Cells. Evid Based Complement Alternat Med 2018;2018:6514705.

26. Song DU, Heo J, Lee SY, et al. Effects of Schizonepeta Spica water extrect on the OVA-induced BALB/c mice model of allergic rhinitis. Kor J Herbal 2015;30:39-46. 